\title{
The Online Temperature Measurement System for Substation Equipment Based on the Internet of Things (IOT)
}

\author{
Xiaoyan $\mathrm{Ma}^{1}$, Hao $\mathrm{Zou}^{2}$ \& Tingting $\mathrm{Xu}^{1}$ \\ ${ }^{1}$ College of Information Engineering, TaiShan Medical University, Taian, Shandong, China \\ ${ }^{2}$ Taian power supply company, Taian, Shandong, China \\ Correspondence: Ma Xiaoyan, College of Information Engineering, TaiShan Medical University, Taian, \\ Shandong, China. Tel: 86-0538-622-9731.E-mail: maxiaoyan81@126.com
}

\author{
Received: March 18, $2014 \quad$ Accepted: April 2, $2014 \quad$ Online Published: May 29, 2014 \\ doi:10.5539/mas.v8n3p217 URL: http://dx.doi.org/10.5539/mas.v8n3p217
}

\begin{abstract}
In order to realize the real-time monitoring of temperature, wireless temperature sensor network is put in the temperature-measuring point in the substation, and then it is transit to the base station through wireless transmission. Finally, by the base station, it is transmit to the management center through the electric power communication network. The temperature management software monitors the temperature timely, visually displays and alarm linkage etc.al. Indoor and outdoor experiments show that the system operates reliably and has good effects, which can accurately realize the online temperature monitoring in a substation.
\end{abstract}

Keywords: temperature monitoring, Internet of Things (IOT) technology, wireless temperature sensing system, visual display

\section{Introduction}

The safe operation of power systems is significant, it tightly related to economic development and economic stability. As the modern power system is developing quickly toward the direction of high voltage, large unit and large capacity, the requirements of reliability is much higher. The temperature characteristic of electrical equipment is the temperature varies slowly at most times, however, when there is an equipment failure (Jiansheng, Da, \& Fan, 2004), a sudden change of temperature may occur. So it is important to perform online temperature monitoring to potential overheat parts. For the substation, Knife contact, cable connectors, copper connections, switch contacts, reactors, capacitors, arc-suppression coil and the equipments and cables within high voltage switchgear is easily overheat because of all kinds of reasons. So it is extremely important to find an online substation temperature monitoring program, which satisfies the requirements of low-priced, easily-conducted and well adapted. The development of the internet of things provides a new way for the substation equipments temperature online monitoring. The internet of thing is a network concept, according to the protocol it connects things to internet to perform intelligent recognition, track, locate, monitor and management.

When power equipment has no faults, the temperature mostly changes gradually and the change is slow (Huang, Li, Wang, \& Fang, 2011); when it has faults, the temperature often changes suddenly. The contingency and emergency of equipment temperature faults decide the fatality of temperature faults. Once the fault appears, there will be heavy loss. The faults caused by overheating of electrical equipment or materials are mostly related to large current, so real-time online monitoring the temperature of large current in the points such as busbar junction (Hwang, Farris, Kompella, \& Chandrasekar, 2003), contact and high voltage cable connector which are easy to overheat is very important.

The main difficulties in online monitoring the temperature anomaly for substation power equipment are: the hot spots of electrical equipment are often in the positions with high potential, making common temperature sensing method under restrictions; electrical equipment has a great number of hot spots to monitor, but because of the limits of sensor isolation, economy and equipment structure, it is impossible to install a great number of complex or expensive temperature sensors (Gao, Liu, \& Zhan, 2011). Therefore, to find an online monitoring temperature solution which is also easy to realize equipment maintenance with low cost and short engineering construction period, as well as good adaptability and extensibility becomes particularly important. 
In recent years, the rapid development of Internet of Things (IOT) technology has provided new path to online monitoring electrical equipment temperature for substation. The term Internet of Things is a concept of network, which connects objects to the internet for the exchange and communication of the information through such information sensing equipments as radio frequency identification, infrared sensor, global poisoning system, laser scanner and so on according to agreed protocol, so as to realize intelligent identification, track, positioning, monitoring and management. The aim of IOT is to connect all the objects to the internet so that the system can automatically real-time identify, locate, track, monitor and trigger corresponding events to the things (Xu \& Nihong, 2005). The online temperature measurement system for substation based on the internet of things (IOT) is one of IOT's important applications in the development of smart grid, which mainly uses digital wireless temperature monitor installation to realize. It can meet the technological needs of online monitoring running temperature for main electrical equipment, power cable, connector, capacitor and electric reactor and provide basic data support for the informatization of total state overhaul (Gong, Yi, Wang, Yue, \& Yu, 2006).

\section{The Design of Online Monitoring Temperature System}

\subsection{System Configuration}

Online monitoring temperature system configuration principle is shown in Figure 1. It is composed by management centre (host machine), data transmission base station and wireless temperature sensor.

Management centre is composed by a host machine based on Windows OS and configuration monitoring software. Host machine is a management platform which integrates various communication protocols and has perfect software functions, and has graphical human-computer interaction interface and complete and steady database. It is mainly used for receiving temperature anomaly alarms sent from thermometry workstation, as well as process, query, statistically analyzing all the data in the system. The management platform not only finishes the temperature management of all the cables, connectors and important equipment in a $220 \mathrm{kV}$ substation, but also implants other management and control functions so as to realize operating multiple management tasks in single platform, which effectively realizes multi-information integration and fusion and reduces users' total cost.

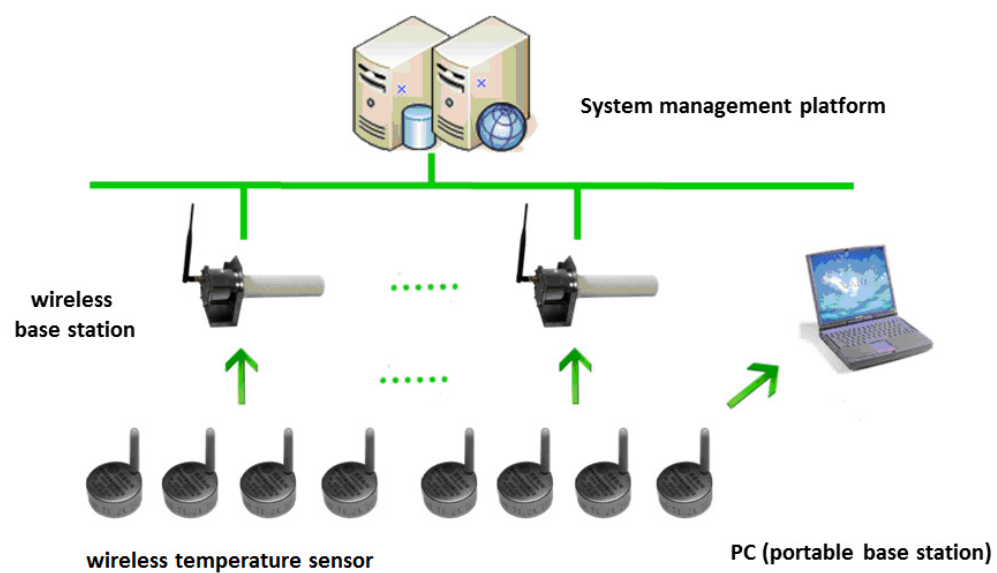

Figure 1. Online monitoring temperature system configuration principle

Data transmission base station automatically receives the data about temperature sent from wireless temperature sensor and uploads the data to thermometry workstation after receiving read command. Because of the limitation of the environment in the site, wireless sensing distance is several hundreds of meters theoretically, but in fact it is only dozens of meters. Each data transmission base station only manages a group of wireless temperature sensor, whose ID has to be configured and saved to terminal flash storage in advance.

Data transmission base station is composed by MCU/RS-485 connector, $2.45 \mathrm{GHz}$ digital RF transceiver, wide input DC/DC stabilized power supply, IP 68 ASA shell, high gain directional antenna and omni-directional antenna. Data transmission base station usually adopts RS- 485 bus to communicate with host machine.

Install a wireless temperature sensor in each point of temperature monitor target. The sensor automatically measures the temperature in the position in every set time limit and outputs the data measured by wireless signal. Each wireless temperature sensor has unique 32 bits ID, which needs to be allocated and record the installation sites of each sensor in practice, and deposited into the configuration file of thermometry workstation. 
Wireless temperature sensor is composed by micro power MCU, digital temperature sensor, $2.45 \mathrm{GHz}$ digital RF transceiver, high-temperature lithium battery, IP168 stainless steel shell and antenna.

\subsection{System Working Principle}

Wireless temperature sensor measures the running temperature in the monitoring point at regular time. Environmental temperature sensor also automatically measures environmental temperature. The temperature data is transmitted to the base station by $2.45 \mathrm{GHz}$ wireless channel, and the base station saves and records the data.

Host machine polls each base station by RS- 485 bus at regular time. Each base station transmits the temperature data received to the host machine. Host machine processes and saves the data.

Through comparisons of the relative temperature rise between equipment and environment, and the temperature rise between indoor and outdoor atmosphere, host machine can analyze possible situation of overheating and issues early warning signal in advance so as to remind manager. Host machine and centralized control station can communicate by Ethernet, or adopt custom temperature model to communicate with host machine in accordance with IEC 61850 Standard, or provide multiple warning signal for public measurement and control screen by relay.

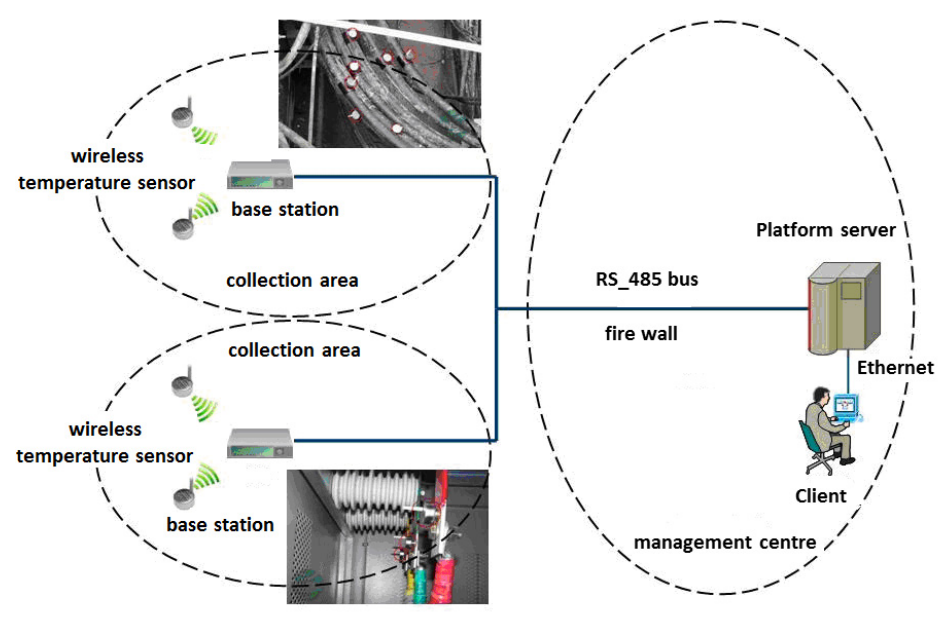

Figure 2. System principle chart

\section{Temperature Monitoring System Software for Substation}

Temperature monitoring system software for substation mainly includes two function modules: data collection server and hot spot monitoring system. Data collection system software obtains real-time data from I/O equipment in the site by I/O device driver and carries out necessary processing of the data. On the one hand, it is shown in the screen of computer visually in graphical form; on the other hand, according to configuration requirements and the commands of operators, it transmits the control data to I/O equipment, and has control of actuator or adjusts control parameter, so as to provide a perfect management function for sample data and the maintenance of database. Hot spot monitoring system is to collect the temperature data about some key equipment in substation and provide display, alarm and printing functions.

Monitoring point map information module shows the specific geographical information about the points monitored, which can realize 3D display, and select a specific point through "location" column; total regional map information module shows all the geographical information about the monitoring points in the substation, which is composed by each small point icon, able to select monitoring point by clicking the small icon in the module; monitoring point data information module shows the specific data information and diagnostic information about the point that is monitored, including ID, Point ID, Point name, Standard code, data, alarm type, alarm grade, area, alarm time and experts' suggestion, whose display can be switchover by the options including alarm information and curve icon.

\section{The Typical Configuration of Online Monitoring Temperature System}

\subsection{The Layout of Data Transmission Base Station}


The first layer assigns seven data transmission base stations (two $10 \mathrm{kV}$ capacitors, four $10 \mathrm{kV}$ switch rooms, one grounding Peterson coil room). The second layer assigns nine data transmission base stations (four $110 \mathrm{kV}$ power distribution equipment rooms, two western walls, three eastern walls)

The sensor in $10 \mathrm{kV}$ switch room is installed in the switch cabinet or busbar bridge. Considering the characteristics of radio wave propagation, four data transmission base stations are lay out. $10 \mathrm{kV}$ capacitor lays out two data transmission base stations; there is no wall between Peterson coil room and switch room, but one data transmission base station.

$110 \mathrm{kV}$ distribution equipment room is open and its equipment density is small, so only laying out 3-4 data transmission base stations reasonably can satisfy the system's requirements. The base station in eastern wall receives signal from main transformer sensors. In consideration of the influence of climate (for example, rain, snow) on communication distance, three base stations are assigned here to improve its reliability. The base station in western wall only receives signal from outside wall bushing sensors. The distance is short, in the premise of ensuring reliability, two data transmission base stations are assigned here.

Indoor installation: base station should select walls and columns far away high-voltage equipment and wire, where the coverage area is wide and the sight of electromagnetic wave is perfect. The height of installation position is usually between 2.5 and 3 meter, where it is convenient to install and maintain the equipment.

Outdoor installation: base station should select walls and towers far away high-voltage equipment and wire, where the coverage area is wide and the sight of electromagnetic wave is perfect. The height of installation position is usually between 2.5 and 3 meter, and on the centralized control room as possible as it can, convenient to lay out the line and shorten the signal line, as well as install and maintain the equipment.

\subsection{The Layout of Wireless Temperature Sensor}

SG-WT100 wireless temperature sensor adopts advanced integration, microminiaturization and equipotential packaging techniques. The project directly installs wireless temperature sensor in cable connector (ingoing and outgoing line of wall bushing, ingoing and outgoing line of current transformer, ingoing and outgoing line of switch, ingoing line of voltage transformer, ingoing and outgoing line of switch blade, outgoing line of switch cabinet, ingoing and outgoing line of transformer, ingoing and outgoing line of Petersen Coil, ingoing line of reactor), switch blade contact, switch contact, copper bar junction point, reactor, Petersen Coil, grounding, capacitor shell, power cable, ingoing and outgoing line of No. $1 \& 2$ transformers and so on so as to realize high-reliability real-time online monitoring temperature, temperature rise and differences. According to the principle that cable connector, switch blade contact, switch contact, copper bar junction point, reactor, Petersen Coil and capacitor shell lay out wireless temperature sensor, 268 points are assigned.

\subsection{The Layout of Host Machine}

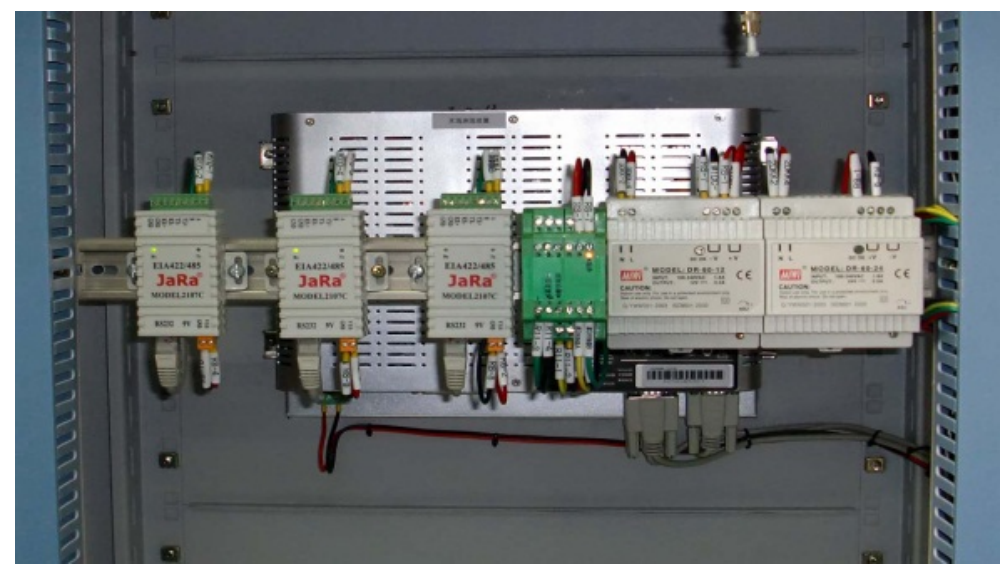

Figure 3. Installation system wiring diagram in the cabinet of industrial control computer

Temperature management software is able to smoothly run in the computer system with 32 bits Windows OS (desk, portable or embedded computer system). In consideration of future extensibility requirements of temperature management software, the project adopts ANOVO $2 \mathrm{U}$ complete industrial control computer, whose configuration is below: RPC-205case, /NOVO-7945mainboard/ BP-05V4 baseboard/ fully-welded 230 industrial power source/Intel dual-core E5300 2.6G CPU/1T hard disk/2G DDRII memory/512M separate video card/Xiba 
RS-232PCI multiport serial card/DVD driver/ optoelectronic packages/AOC 19 inches of liquid crystal display, which satisfy the hardware requirements of current temperature management software and provide sufficient hardware redundancy for future system function extensions. The system wiring diagram in the cabinet of industrial control computer is shown in Figure3.

\subsection{The Solution That Host Machine Polls Each Base Station}

Wireless temperature sensor sends data to base station regularly. Wireless base station and wireless temperature sensor constitute wireless temperature sensing network through $2.4 \mathrm{GHz}$ WSN aerial wireless communication protocol and finish initial reception, storage and upload of the data. The data bearing network from wireless base station to main control room uses 485 bus. Host machine polls each base station by RS- 485 bus regularly. Each base station transmits temperature data it receives to host machine. Host machine processes and saves the temperature data. After management centre receive the data, background software system finishes the receipt, storage and visual display, and has a deep mining of the data. Through various expert models, the function such as intelligent analysis and early warning of monitoring contents is realized, as well as the combination with other application system, so as to realize the sharing and mutual communication of the data between systems.

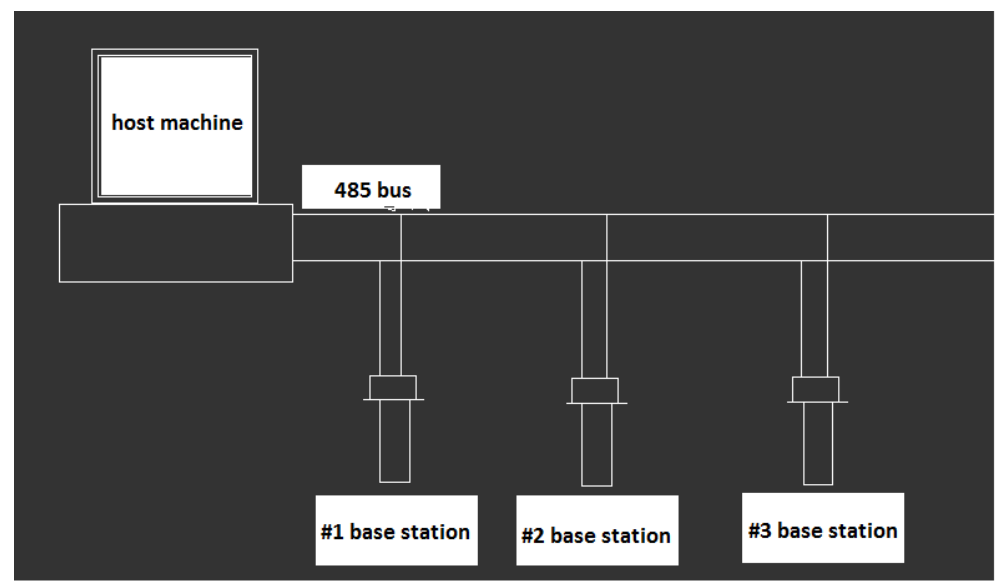

Figure 4. The diagram that host machine polls base station

That host machine polls each base station adopts unicast polling. No specific information is sent to host machine to realize polling. Uplink can allocate sufficient bandwidth that is used for the terminal sends request in mapping information. If the terminal does not need send request, the time slot corresponding to the allocation should be filled according to the protocol. If the terminal has an active UGS link and sufficient bandwidth, it should not be unicast polling, only when the terminal is set up in PM bit in the frame header of UGS link, which is able to avoid unicast polling all the terminal and thus save bandwidth. What needs to notice is that unicast polling for single terminal is usually realized by data authorization mechanism that allocates basic CiD aimed at the terminal.

\section{Conclusion}

The temperature monitoring system for substation based on IOT technology is a reform of power equipment temperature monitoring method. Compared with traditional thermometry methods such as wax sheet or infrared measurement, it not only saves a great deal of manpower and material resources, but also has the advantages such as high accuracy, rapid reaction speed, easy installation, small in size, all-weather, real-time, online, no failure to report and strong immunity from interference, so it is of great engineering significance.

The program of temperature online monitoring is to place wireless sensor networks at temperature monitoring point. Through the wireless network temperature data is transmitted to base station. Then the base station transmits data to management center and the temperature management software will realize online monitoring, visible display and linkage alarm. According to operation temperature, relative temperature and temperature difference the state of equipments can be judged.

This paper compares some different methods of substation temperature monitoring. Through the comparison the paper presents the temperature monitoring method based on the technique of the internet of things. In this paper, the method presented is studied and analyzed, research program, typical configuration and the function is given 
too. The substation temperature online monitoring program is an important application in the smart grid. This program is basically realized by digital wireless temperature monitoring equipment, it is able to achieve online temperature monitoring on all major equipments of substation, and is also able to provide data support to condition-based maintenance information. The program presented in this paper is an evolution of electrical equipment temperature monitoring. Compared to traditional methods, it is low-priced, easily-conducted and well adapted, so it is of great engineering significance.

\section{Acknowledgments}

Supported by the Tai'an Science and Technology Bureau (Project: Research on intelligent substation data integration and visualization management) and China Society of Logistics (2014).

\section{References}

Gao, Z., Liu, X., \& Zhan, T. (2011). The research of tempration control system based on type-k thermocouple. Machinery Design \& Manufacture, 4, 5. $\quad$ Retrieved from http://en.cnki.com.cn/Article_en/CJFDTOTAL-JSYZ201104005.htm

Gong, X. F., Yi, H. G., Wang, C. S., Yue, S. F., \& Yu, B. (2006). Research on Temperature Monitoring of Isolators in HV Switchgear. Paper presented at the Zhongguo Dianji Gongcheng Xuebao (Proceedings of the Chinese Society of Electrical Engineering). Retrieved from http://en.cnki.com.cn/Article_en/CJFDTOTAL-ZGDC200601029.htm

Huang, X., Li, X., Wang, Y., \& Fang, S. (2011). An online temperature monitoring system of substation based on Zigbee wireless network. Paper presented at the Electrical and Control Engineering (ICECE), 2011 International Conference on. http://dx.doi.org/10.1109/ICECENG.2011.6057255

Hwang, J., Farris, T. N., Kompella, S., \& Chandrasekar, S. (2003). Measurement of temperature field in surface grinding using infra-red (IR) imaging system. Journal of Tribology, 125(2), $377-383$. http://dx.doi.org/10.1115/1.1537748

Jiansheng, L., Da, F., \& Fan, Z. (2004). A novel method for remote on-live temperature detection of substation high-voltage contacts. Automation of Electric Power Systems, 4, 13. Retrieved from http://en.cnki.com.cn/Article_en/CJFDTOTAL-DLXT200404013.htm

$\mathrm{Xu}$, Y., \& Nihong, G. (2005). The on-line detector of temperature for the contact inside the H. V. Switchgear. High Voltage Apparatus, 41(2), 139-140. http://dx.doi.org/10.3969/j.issn.1001-1609.2005.02.019

\section{Copyrights}

Copyright for this article is retained by the author(s), with first publication rights granted to the journal.

This is an open-access article distributed under the terms and conditions of the Creative Commons Attribution license (http://creativecommons.org/licenses/by/3.0/). 